\title{
Optimized Extract from Corylopsis coreana Uyeki (Hamamelidaceae) Flos Inhibits Osteoclast Differentiation
}

\author{
Yongjin Lee, ${ }^{1}$ Jung-Eun Kim, ${ }^{2}$ Kwang-Jin Kim, ${ }^{1}$ Seung-Sik Cho $(1),{ }^{2}$ and Young-Jin Son $\mathbb{1}^{1}$ \\ ${ }^{1}$ Department of Pharmacy, Sunchon National University, Jeonnam, Suncheon 57922, Republic of Korea \\ ${ }^{2}$ Department of Pharmacy, College of Pharmacy, Mokpo National University, Muan, Jeonnam 58554, Republic of Korea
}

Correspondence should be addressed to Seung-Sik Cho; sscho@mokpo.ac.kr and Young-Jin Son; sony@sunchon.ac.kr

Received 15 September 2017; Revised 23 December 2017; Accepted 30 January 2018; Published 6 March 2018

Academic Editor: Michał Tomczyk

Copyright (C) 2018 Yongjin Lee et al. This is an open access article distributed under the Creative Commons Attribution License, which permits unrestricted use, distribution, and reproduction in any medium, provided the original work is properly cited.

\begin{abstract}
Osteoporosis is a metabolic disorder that decreases the stability against fractures of the spine, femur, and radius by weakening the strength and integrity of bones. Receptor activator of nuclear factor-kappa B ligand signaling ultimately activated nuclear factoractivated $\mathrm{T}$ cells $\mathrm{cl}$, a major transcription factor for osteoclast formation. This study researched the effects of Corylopsis coreana (C. coreana) Uyeki flos extracts on the antiosteoclastic potential of macrophages and the phytochemicals contained therein. The alcoholic extract of $C$. coreana Uyeki flos inhibited the differentiation of osteoclast. We carried out the experiments of the pattern of differentiation of osteoclasts based on the alcoholic percentage of extracts. Among them, $80 \%$ alcoholic extract showed the highest inhibitory effect. The alcoholic extract was composed of phytochemicals such as bergenin, quercetin, and quercitrin. This extract inhibited not only mRNA expression levels of NFATc1, osteoclast-associated receptor (OSCAR), cathepsin K, and tartrate-resistant acid phosphatase (TRAP), but also the translational expression of NFATcl. The inhibitory effect for osteoclast differentiation of the alcoholic extract was confirmed using the resorption pit assay. This is the first scientific report of the antiosteoclastic effects of $C$. coreana Uyeki flos extract, which can be applied therapeutically for the treatment of osteoporosis.
\end{abstract}

\section{Introduction}

Osteoporosis is a metabolic disease in which the bone integrity and strength are weak due to qualitative changes in the bone microstructure, which decreases the stability against fractures of the spine, femur, and radius. In diseases, such as osteoporosis, the number of osteoclasts is increased resulting in a decrease in bone density. Moreover, in inflammatory conditions, for example, rheumatoid arthritis and periodontitis, osteoclast formation is activated with concomitant bone destruction. The differentiation of osteoclasts is regulated by receptor activator of nuclear factor-kappa B ligand (RANKL), a cytokine essential for the bone metabolism [1]. The interaction between RANK and RANKL activates the continuity of intracellular molecular phenomena directly, leading to osteoclastogenesis. The osteoclast differentiation pathway by RANKL includes NF- $\kappa \mathrm{B}$, mitogen-activated protein kinase (MAPK), including c-Jun N-terminal kinase (JNK), extracellular signal-regulated kinase (ERK), and p38 [2]. This signal transduction can interact with nuclear factor-activated
T cells cl (NFATc1), a master regulator of osteoclastogenesis, leading to translocation into the nucleus [3]. NFATc1, a member of the transcription factors NFAT family, is increased significantly during RANKL-induced osteoclastogenesis [4].

C. coreana Uyeki flos belongs to the Hamamelidaceae (witch hazel) family and is cultured as a medicinal plant in China and South Korea [5]. C. coreana Uyeki flos has been utilized as a traditional medicine to treat chills, nausea, dizziness, and heart pounding $[5,6]$ but there are few scientific reports of $C$. coreana Uyeki flos. In a previous study, some compounds from the leaves of C. coreana Uyeki flos were extracted and identified. It was discovered that they had antioxidative and antiproliferative components [5]. Recently, the authors identified flavonoids and isocoumarin in the flower extract of $C$. coreana Uyeki flos and reported their antimicrobial, antioxidant, and antihyperuricemic activity [7, 8]. On the other hand, to the best of the authors' knowledge, there is a paucity of reports on the antiosteoclastic activity of C. coreana Uyeki flos. 
Therefore, this study examined the osteoclastic activity of the alcoholic extracts of $C$. coreana Uyeki flos and identified the active constituents responsible for the antiosteoclastic effects using the relevant in vitro systems. This study could provide a basic information for the complementary and alternative herbal medicines for the treatment of osteoporosis and its related diseases.

\section{Materials and Methods}

2.1. Plant Materials. Samples of C. coreana Uyeki flos were collected in May 2013 near Jogye Mountain, in Jeonnam Province, Korea. A voucher specimen (MNUCSS-CC-01) was deposited in Mokpo National University (Muan, Republic of Korea). The air-dried and powdered samples of C. coreana Uyeki flos (10 g) were extracted twice with diverse percentages of ethanol $(100 \mathrm{~mL})$ at RT for 3 days. After filtration, the resulting ethanol solution was evaporated, freeze-dried, and stored at $-50^{\circ} \mathrm{C}$. The crude extract was resuspended in ethanol and filtered using a $0.4 \mu \mathrm{m}$ membrane filter. All samples were used for the optimization of extract and in vitro experiments.

2.2. Constituent Purification and Profiling by High-Performance Liquid Chromatography (HPLC). The air-dried, powdered extract of $C$. coreana Uyeki flos was sonicated for $3 \mathrm{hrs}$ with ethanol. After filtration, the ethanol was evaporated and suspended in distilled water and then defatted with an $n$ hexane, ratio of $1: 1(\mathrm{v} / \mathrm{v})$. The aqueous layer was partitioned with ethyl acetate $(2 \times 200 \mathrm{~mL})$. The evaporation residue $(2 \mathrm{~g})$ was subjected to flash silica gel chromatography, using an $n$-hexane: EtOAc solvent system $(9: 1 \sim 3: 1)$, to afford 10 fractions. Fractions 2, 3, and 4 were subjected to further RPC18 silica gel chromatography, using an acetonitrile: water $(10: 100)$ eluent system, to afford bergenin (BER), quercitrin (QCIT), and quercetin (QCT). The compounds were further purified by preparative thin layer column chromatography for analytically acceptable purity. Constituent-profiling of the $C$. coreana Uyeki flos samples by HPLC analysis was performed as previously described [8]. All analysis was conducted using an Alliance 2695 HPLC system (Waters; Millford, MA, USA) equipped with a photodiode array detector. The analytical column used was an Agilent Zorbax extended C18 column $(5 \mu \mathrm{m}, 150 \mathrm{~mm} \times 5 \mathrm{~mm})$ with a mobile phase consisting of solvents A (acetonitrile) and B (water containing $0.2 \%$ phosphoric acid) and employing gradient elution (from 10/90 to $100 / 0, \mathrm{v} / \mathrm{v}$ ) at a flow rate of $0.8 \mathrm{~mL} / \mathrm{min}$. The column temperature was maintained at $25^{\circ} \mathrm{C}$, and the detection wavelength was set to $350 \mathrm{~nm}$ for BER, QCT, and QCIT. The mobile phase was filtered through a $0.22 \mu \mathrm{m}$ filter and degassed.

2.3. Cell Cultures and Osteoclast Differentiation. This study was conducted in strict accordance with the recommendations contained in the Standard Protocol for Animal Study of Sunchon National University. The protocol was approved by the Sunchon National University Institutional Animal Care and Use Committee (SCNU IACUC; Permission number SCNU IACUC 2016-07). All cells were cultured in a $37^{\circ} \mathrm{C}$ and $5 \% \mathrm{CO}_{2}$ incubator. Bone marrow-derived macrophages (BMMs) were derived from unfractionated bone marrow cells (BMCs). The BMCs were isolated from the tibia and femur of 5-week-old male ICR mice $(n=2$ : Damool Science, KR) by flushing with $\alpha$-minimum essential medium ( $\alpha$-MEM; Invitrogen Life Technologies, CA, USA) containing $100 \mathrm{U} / \mathrm{mL}$ penicillin/streptomycin (Invitrogen, CA, USA). The cells were incubated on a Petri dish in $\alpha$-MEM supplemented with $10 \%$ fetal bovine serum (FBS; Invitrogen Life Technologies, CA, USA) and $100 \mathrm{U} / \mathrm{mL}$ penicillin/streptomycin (10\% $\alpha$-MEM) with $30 \mathrm{ng} / \mathrm{mL}$ of the mouse recombinant macrophage colony-stimulating factor (M-CSF; PEPROTECH, NJ, USA). After 3 days, the cells attached to Petri dishes were obtained as BMMs. The BMMs were plated at a density of $1 \times 10^{4}$ cells/well in a 96-well tissue culture plate in $10 \% \alpha$-MEM and cultured with $10 \mathrm{ng} / \mathrm{mL}$ of the mouse recombinant receptor activator of nuclear factor$\kappa \mathrm{B}$ ligand (RANKL; R\&D Systems, MN, USA) and $30 \mathrm{ng} / \mathrm{mL}$ $\mathrm{M}$-CSF for 4 days in the presence or absence of $C$. coreana Uyeki flos.

\subsection{Tartrate-Resistant Acid Phosphatase (TRAP) Staining} Assay. The cells were washed with PBS and fixed with $3.7 \%$ formalin for $5 \mathrm{~min}$. After washing with PBS, the cells were permeabilized with $0.1 \%$ Triton $\mathrm{X}-100$ for $10 \mathrm{~min}$. And, then, they were washed, and stained for $10 \mathrm{~min}$ at $37^{\circ} \mathrm{C}$ in the dark with a TRAP solution containing Fast Garnet GBC, sodium nitrite, naphthol AS-BI phosphoric acid, acetate, and tartrate (Sigma-Aldrich, MO, USA). The TRAP+-MNCs (nuclei $\geq 3$ ) were counted as mature osteoclasts.

2.5. Cytotoxicity Assay. The BMMs were cultured at a density of $1 \times 10^{4}$ cells/well on a 96-well plate in triplicate with MCSF $(30 \mathrm{ng} / \mathrm{mL})$ and C. coreana Uyeki flos. The cell viability was evaluated using a CCK-8 kit (Dojindo Molecular Technologies, Japan) according to the manufacturer's protocol.

2.6. Real-Time PCR Analysis. The BMMs were plated at $3.5 \times 10^{4}$ cells/well in a 6 -well plate and cultured with $10 \mathrm{ng} / \mathrm{mL}$ RANKL and $30 \mathrm{ng} / \mathrm{mL} \mathrm{M}$-CSF for $0,1,2$, and 3 days in the presence or absence of C. coreana Uyeki flos. The primers for real-time PCR were designed (Table 1) using the Primer3 design program. The total RNAs were isolated with TRIzol reagent (Invitrogen, CA, USA), and $1 \mu \mathrm{g}$ of RNAs was reverse-transcribed with the M-MLV cDNA Synthesis kit (Enzynomics, KR) according to the manufacturer's protocol. Quantitative PCR was accomplished using a TOPreal qPCR 2x PreMIX (Enzynomics, KR) and Real-Time PCR detection system (Bio-Rad, CA, USA). All tests were run in triplicate and normalized to the housekeeping gene GAPDH.

2.7. Western Blot Analysis. The cells were washed with phosphate-buffer saline (PBS) and homogenized with a lysis buffer containing $20 \mathrm{mM}$ Tris-HCl, pH 7.5, 1\% v/v Igepal CA630, $150 \mathrm{mM} \mathrm{NaCl}, 1 \mathrm{mM}$ sodium fluoride (Sigma, USA), $1 \mathrm{mM}$ sodium orthovanadate (Sigma, USA), $0.5 \mathrm{mM}$ phenylmethylsulfonyl fluoride (Sigma, USA), $1 \mathrm{mM}$ DTT, $2 \mathrm{mM}$ EDTA, $10 \mu \mathrm{g} / \mathrm{mL}$ aprotinin (Sigma, USA), $5 \mu \mathrm{g} / \mathrm{mL}$ leupeptin 
TABLE 1: Primer sequences used in this study.

\begin{tabular}{lcr}
\hline Gene of interest & Direction & Primer sequence $\left(5^{\prime}-3^{\prime}\right)$ \\
\hline TRAP & Sense & GATGACTTTGCCAGTCAGCA \\
& Antisense & ACATAGCCCACACCGTTCTC \\
NFATc1 & Sense & GGGTCAGTGTGACCGAAGAT \\
& Antisense & GGAAGTCAGAAGTGGGTGGA \\
Cathepsin $\mathrm{K}$ & Sense & GGCCAACTCAAGAAGAAAAC \\
& Antisense & GTGCTTGCTTCCCTTCTGG \\
OSCAR & Sense & CTGCTGGTAACGGATCAGCTC \\
& Antisense & CCAAGGAGCCAGAACCTT \\
GAPDH & Sense & AACTTTGGCATTGTGGAAGG \\
& Antisense & ACACATTGGGGGTAGGAACA \\
\hline
\end{tabular}

(Sigma, USA), and $2 \mu \mathrm{g} / \mathrm{mL}$ pepstatin (Sigma, USA). The lysates were centrifuged at $12,000 \mathrm{~g}$ for $15 \mathrm{~min}$ at $4^{\circ} \mathrm{C}$. The protein concentration was determined using a DC Protein Assay (Bio-Rad, CA, USA). The protein extracts were subjected to $10 \%$ sodium dodecyl sulfate-polyacrylamide gel electrophoresis (SDS-PAGE) (20 $\mu$ g per lane) and transferred to a polyvinylidene difluoride (PVDF) membrane (Millipore, USA). After blocking with $5 \%$ skim milk the membranes were incubated overnight at $4^{\circ} \mathrm{C}$ with the primary antibodies (NFATcl and actin antibodies from Santa Cruz Biotechnology, CA, USA). After washing, the membranes were incubated with the horseradish peroxidase- (HRP-) conjugated secondary antibody (Santa Cruz Biotechnology, CA, USA) for $2 \mathrm{hrs}$ at room temperature. The antibody blots were developed using a MicroChemi 4.2 (DNR Bio-Imaging System, Jerusalem, Israel) and Super-Signal West Pico/Femto Chemiluminescent Substrate (Pierce Chemical, IL, USA).

2.8. Resorption Pit Analysis. To analyze the surface for pit formation, the BMMs were plated at $6 \times 10^{6}$ cells/well in a 24-well tissue culture plate and cultured with the conditions of $30 \mathrm{ng} / \mathrm{mL} \mathrm{M}-\mathrm{CSF}$ in a negative/positive (without/with $10 \mathrm{ng} / \mathrm{mL}$ RANKL) and the presence (10 and $30 \mu \mathrm{g} / \mathrm{mL})$ of $80 \%$ C. coreana Uyeki flos for 4 days. The media were discarded and $100 \mu \mathrm{L}$ of $10 \%$ bleach solution was changed on day 4 . And cells were incubated for $5 \mathrm{~min}$ at RT. The plate was washed twice with distilled water and dried at RT for about $4 \mathrm{hrs}$. The generated pits were observed using a microscope at $100 \mathrm{x}$ magnification.

2.9. Statistical Analysis. The proteins and RNA from C. coreana Uyeki flos and control groups were analyzed to determine the differences in the levels of expression using Student's $t$ test. Probability $(P)$ values less than 0.05 were considered significant $\left({ }^{*} P<0.05,{ }^{* *} P<0.01\right.$, and $\left.{ }^{* * *} P<0.001\right)$.

\section{Results}

3.1. C. coreana Uyeki Flos Inhibits the Differentiation of BMMs into Osteoclasts. To examine the effects of $C$. coreana Uyeki flos on RANKL-induced osteoclast differentiation, we performed in vitro osteoclast differentiation. The BMMs were treated with the vehicle $(0.1 \%$ DMSO) as a control and the C. coreana Uyeki flos extract and then mixed with $30 \mathrm{ng} / \mathrm{mL}$ M-CSF and $10 \mathrm{ng} / \mathrm{mL}$ RANKL for 4 days. We conducted the TRAP staining to determine the most effective alcohol concentrations; the inhibitory effect of osteoclastogenesis was highest with $80 \%$ alcohol extract (Figure 1(a)). We performed the experiment on the optimal amounts of samples to inhibit osteoclast differentiation. The $80 \%$ extract of C. coreana Uyeki flos at a concentration above $10 \mu \mathrm{g} / \mathrm{mL}$ inhibited the osteoclast differentiation. Its inhibitory effect appeared as the plateau above $30 \mu \mathrm{g} / \mathrm{mL}$ (Figures 1(b) and 2(a)). Moreover, the $80 \%$ extract of C. coreana Uyeki flos at a concentration above $10 \mu \mathrm{g} / \mathrm{mL}$ inhibited the differentiation of TRAP $^{+}$-osteoclasts. The number of TRAP ${ }^{+}$-MNCs (nuclei $\geq$ 3) was decreased dose-dependently by C. coreana Uyeki flos (Figure 2(b)).

3.2. C. coreana Uyeki Flos Had No Cytotoxic Effect. The toxic effects of C. coreana Uyeki flos on the BMMs were identified. The BMMs were incubated with C. coreana Uyeki flos-treated medium containing $30 \mathrm{ng} / \mathrm{mL}$ M-CSF for 3 days and the cell viability was measured by the CCK-8 kit (Figure 2(c)). C. coreana Uyeki flos at concentrations less than $30 \mu \mathrm{g} / \mathrm{mL}$ showed no cytotoxicity to the BMMs.

3.3. Main Components Identified from the $80 \%$ Alcoholic Extracts. Table 2 listed total flavonoids, phenolics, and the contents of active constituents of phytochemicals. The $80 \%$ alcoholic extract of $C$. coreana Uyeki flos was composed of $269 \mathrm{mg}$ of total phenolics, $16 \mathrm{mg}$ of total flavonoids, and some phytochemicals, such as bergenin (BER), quercetin (QCT), and quercitrin (QCIT). The contents of BER, QCT, and QCIT were $17.01,1.50$, and $0.05 \%(\mathrm{w} / \mathrm{w})$, respectively, in the $80 \%$ alcoholic extract.

3.4. C. coreana Uyeki Flos Suppresses RANKL-Induced Gene Expression. Real-time PCR analysis was performed to confirm the effects of $C$. coreana Uyeki flos on osteoclast formation and the expression of transcription factors and osteoclast-specific markers. C. coreana Uyeki flos reacted with RANKL and inhibited the mRNA expression of OSCAR and NFATcl, which are important transcription factors 

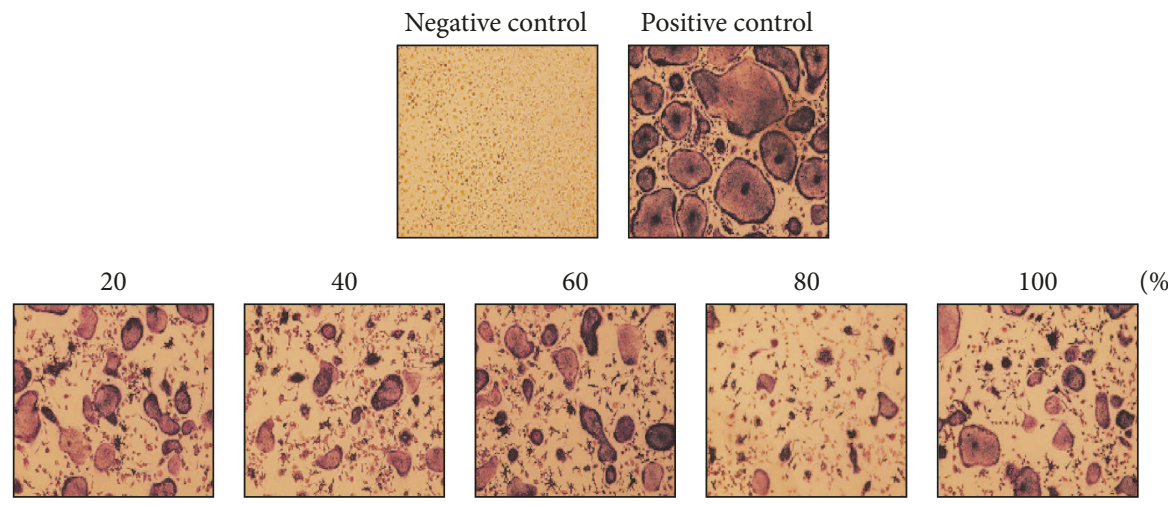

(\%)

Sample conc.

$(30 \mu \mathrm{g} / \mathrm{mL})$

(a)
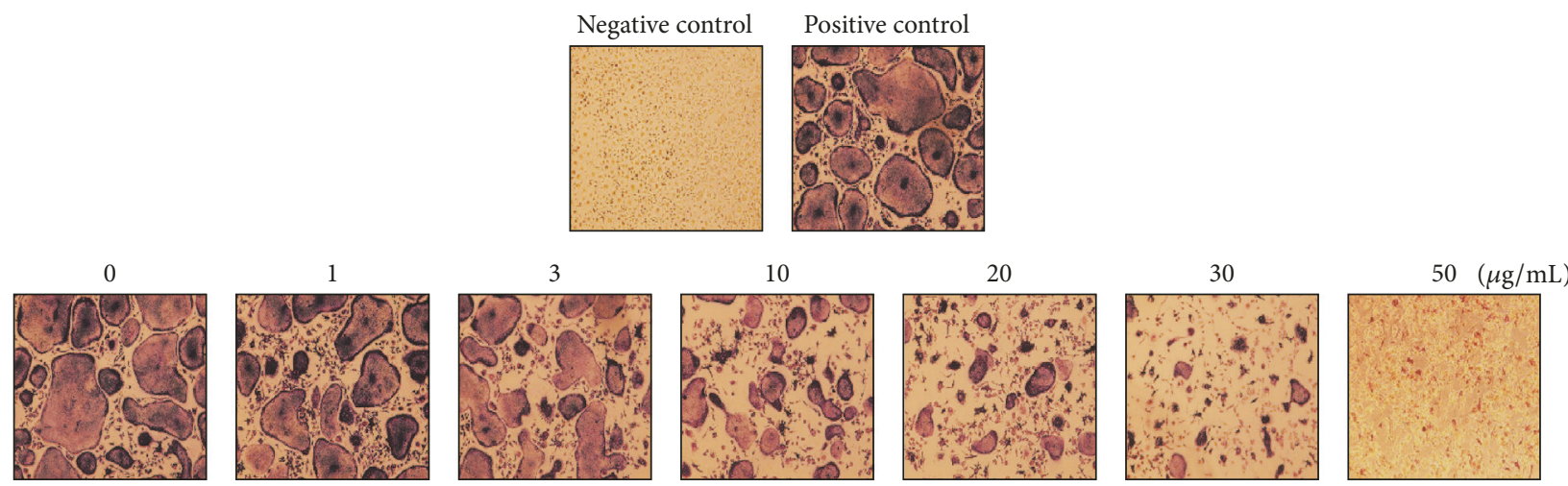

(b)

FIGURE 1: Osteoclast differentiation was inhibited by C. coreana Uyeki flos extracts. BMMs were cultured for 4 days in $0.1 \%$ DMSO (control vehicle) or the concentration $30 \mu \mathrm{g} / \mathrm{ml}$ of C. coreana Uyeki flos extract with RANKL ( $10 \mathrm{ng} / \mathrm{mL})$ and M-CSF ( $30 \mathrm{ng} / \mathrm{ml})$. The inhibitive patterns of osteoclast differentiation based on the alcoholic percentage of extracts (a). The inhibitive patterns of osteoclast differentiation based on the concentration of the $80 \%$ alcoholic extract of C. coreana Uyeki flos (b).

TABle 2: Total flavonoids, phenolics, and contents of active constituents of the main phytochemicals identified from the $80 \%$ ethanolic extract of C. coreana Uyeki flos $(n=5)$.

\begin{tabular}{lc}
\hline Constituent & Content \\
\hline Total flavonoids & $16.43 \pm 0.09(\mathrm{mg} / \mathrm{g}$ eq.quercetin) \\
Total phenolics & $269.37 \pm 98.72(\mathrm{mg} / \mathrm{g}$ eq.gallic acid $)$ \\
Bergenin & $17.01 \pm 0.08 \%(\mathrm{w} / \mathrm{w})$ \\
Quercetin & $1.5 \pm 0.007 \%(\mathrm{w} / \mathrm{w})$ \\
Quercitrin & $0.05 \pm 0.002 \%(\mathrm{w} / \mathrm{w})$ \\
\hline
\end{tabular}

involved in osteoclast differentiation (Figures 3(a) and 3(b)). In addition, the mRNA expression levels of cathepsin $K$ and TRAP involved in NFATcl expression were inhibited significantly in osteoclast differentiation (Figures 3(c) and $3(\mathrm{~d}))$.

3.5. C. coreana Uyeki Flos Suppresses RANKL-Induced Protein Expression of NFATc1. The effect of C. coreana Uyeki flos on the protein level of NFATcl, a key regulator of osteoclast differentiation, was determined by western blotting. RANKL increased the protein expression of NFATc1 dramatically over time, but the level of NFATc1 protein expression was reduced significantly after exposure to $C$. coreana Uyeki flos (Figure 4). This result suggested that C. coreana Uyeki flos inhibited the protein expression of NFATcl, which is key element in the osteoclast formation process.

3.6. C. coreana Uyeki Flos Suppresses RANKL-Induced Resorptive Activity. To confirm the inhibitory activity of the alcoholic extract of C. coreana Uyeki flos in osteoclast differentiation, we performed the resorption pit assay that can resorb the mineralization matrix and indicate the ability to form resorption pits on the surface. This experiment showed a lot of pits produced by human osteoclast precursor cells on the Corning Osteo Assay Surface in a positive well, compared with a negative well (Figures 5(a) and 5(b)). It was confirmed that the Corning Osteo Assay Surface was a good substrate for the analysis of osteoclast functional activity. When the alcoholic extract of $C$. coreana Uyeki flos was added, the number of resorptive pits was decreased in proportion to the adding amounts of the extract (Figures 5(c) and 5(d)). This result demonstrated that the alcoholic extract of C. coreana Uyeki flos inhibited the resorptive activity of osteoclast induced by RANKL. 


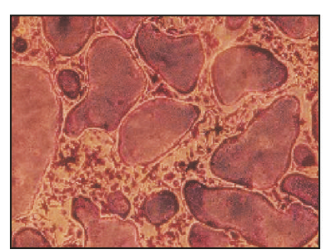

RANKL $80 \%$ extract

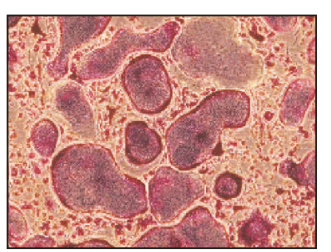

$+$

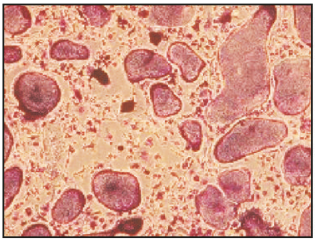

$+$

10

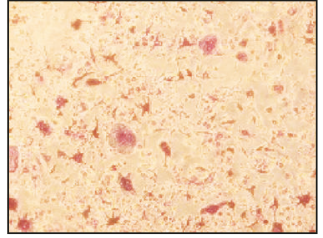

$+$

30
$(10 \mathrm{ng} / \mathrm{mL})$

$(\mu \mathrm{g} / \mathrm{mL})$

(a)

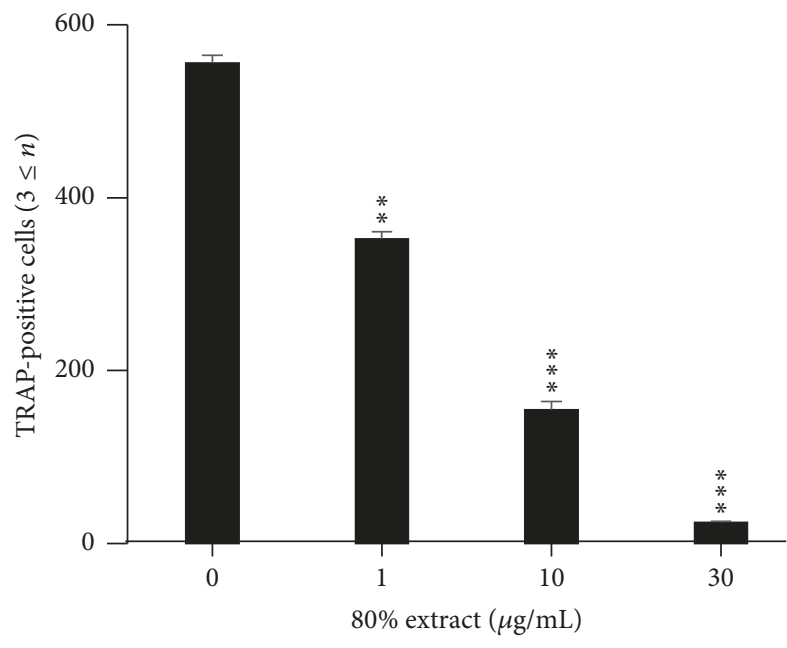

(b)

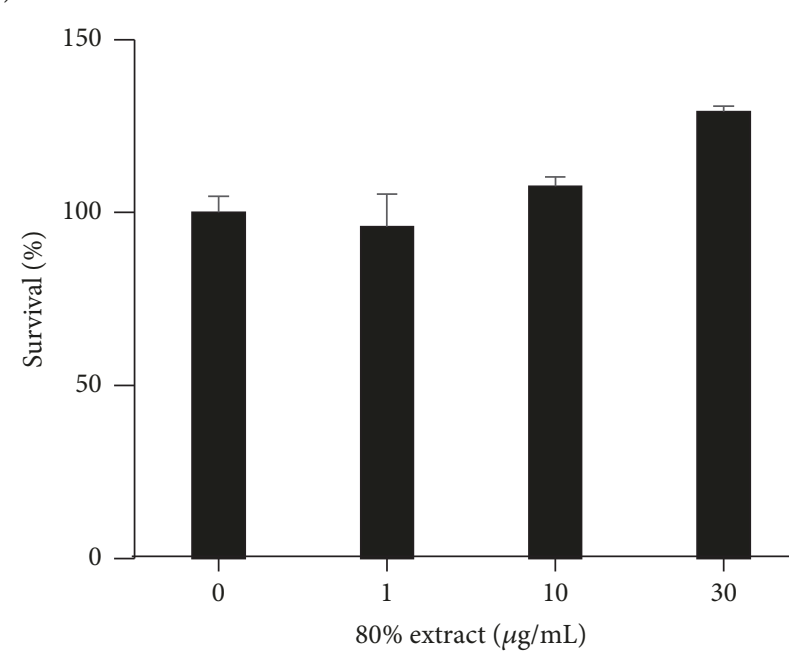

(c)

FIGURE 2: RANKL-mediated osteoclastogenesis was suppressed by C. coreana Uyeki flos extracts, and the extracts had no cytotoxic effect. (a) BMMs were cultured for 4 days in $0.1 \%$ DMSO (control vehicle) or the indicated concentrations of C. coreana Uyeki flos extract with RANKL $(10 \mathrm{ng} / \mathrm{mL})$ and M-CSF (30 ng/ml). Multinucleated cells were fixed (3.7\% formalin), permeabilized (0.1\% Triton X-100), and stained with the TRAP solution. (b) Mature TRAP-positive multinucleated osteoclasts (MNCs) were photographed under an optical microscope. TRAPpositive MNCs (nuclear number $>3$ ) were counted. ${ }^{* *} P<0.01$; ${ }^{* * *} P<0.001$. (c) The effect of $C$. coreana Uyeki flos extracts on the viability of BMMs was measured by the CCK- 8 assay.

\section{Discussion}

Imbalances between osteoblasts and osteoclasts induced by various factors cause excessive bone resorption, which is particularly characteristic of osteoporosis. In the bone marrow, osteoclastic bone cells differentiate from hematopoietic stem cells $[9,10]$. Osteoclastic cells are often differentiated in vitro when BMMs are treated with RANKL and M-CSF [11]. Activation of the RANKL-induced NF-kB and MAPK pathways is an important mechanism for osteoclastogenesis [12-14]. In this study, the alcoholic extracts derived from C. coreana Uyeki flos inhibited osteoclast differentiation from the BMMs and downregulated the expression of the marker genes and involved protein by blocking the RANKLinduced signaling pathway [15]. In addition, we confirmed that the osteoclast suppressed by $C$. coreana Uyeki flos had a resorptive activity using the resorption pit assay. These results suggested that $C$. coreana Uyeki flos could be used as a therapeutic agent to inhibit bone breakdown in osteolytic bone diseases such as osteoporosis.

A number of flavonoids and phenolic compounds possess antiosteoclastic activity [16]. Therefore, the ethanol extraction process was optimized with respect to the total flavonoids and total phenolics. In a previous study, the $80 \%$ alcoholic extract showed the optimal flavonoid and phenolic content $[7,8]$. Therefore, according to the results of a previous study and the findings of the current study, the $80 \%$ alcoholic extract was selected for further in vitro studies. A previous study showed that BER, QCT, and QCIT were the main bioactive phytochemicals identified from C. coreana Uyeki flos [7]. The $80 \%$ alcoholic extract exhibited the highest antiosteoclastic activity among all extracts examined (Figures 1 and 2). Therefore, these constituents were analyzed in the $80 \%$ alcoholic extract prepared in the present study. BER was one of the major compounds in the $80 \%$ alcoholic extract of C. coreana Uyeki flos. Nazir et al. reported that BER inhibits the formation of proinflammatory helper T cell type 1 cytokines (TNF- $\alpha$, IFN- $\gamma$, and IL-2), while it potentiates the anti-inflammatory helper T cell type 2 cytokines (IL-5 and IL4) in the arthritic mice model [17]. QCT and QCIT appear to be important active compounds in the $80 \%$ alcoholic extract. Oliveira et al. reported that QCT did not affect the cell viability and decreased osteoclast formation compared with a control and downregulated NF- $\kappa$ B pathway. They suggested that QCT might be inhibitor of osteoclast differentiation under inflammatory conditions (LPS-induced) through attenuation 


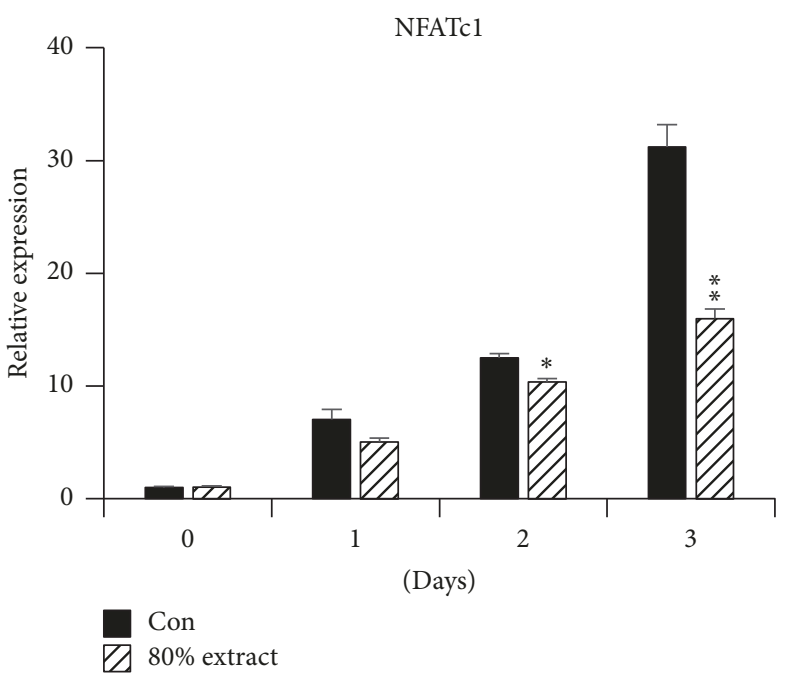

(a)

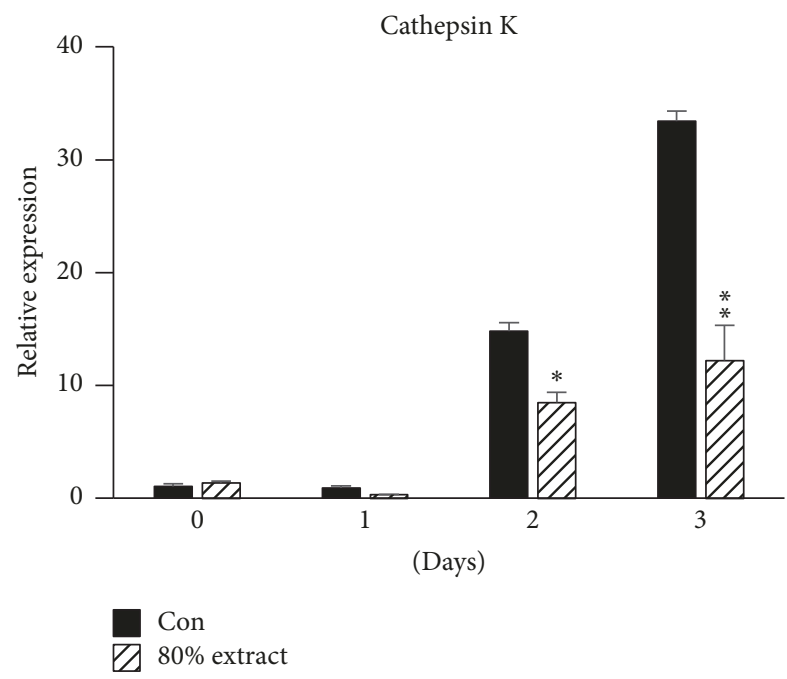

(c)

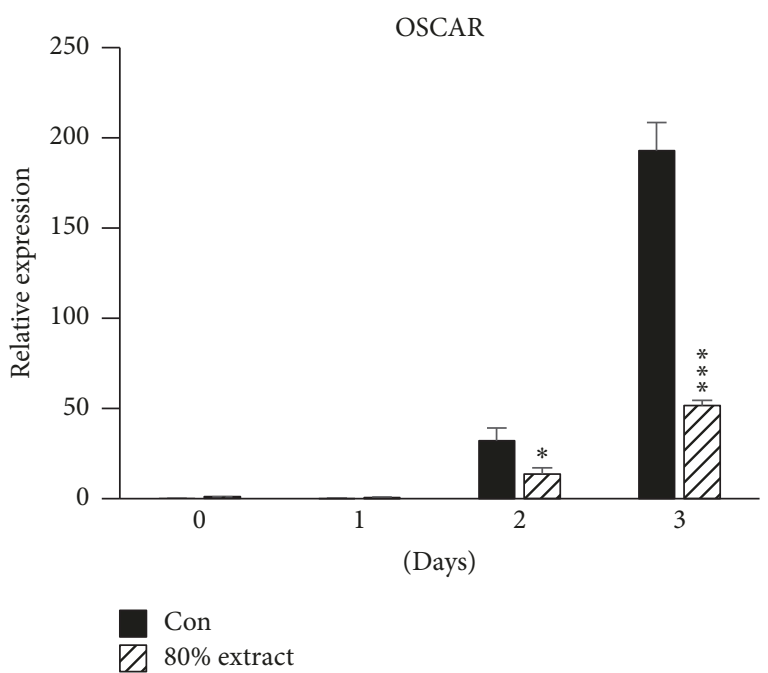

(b)

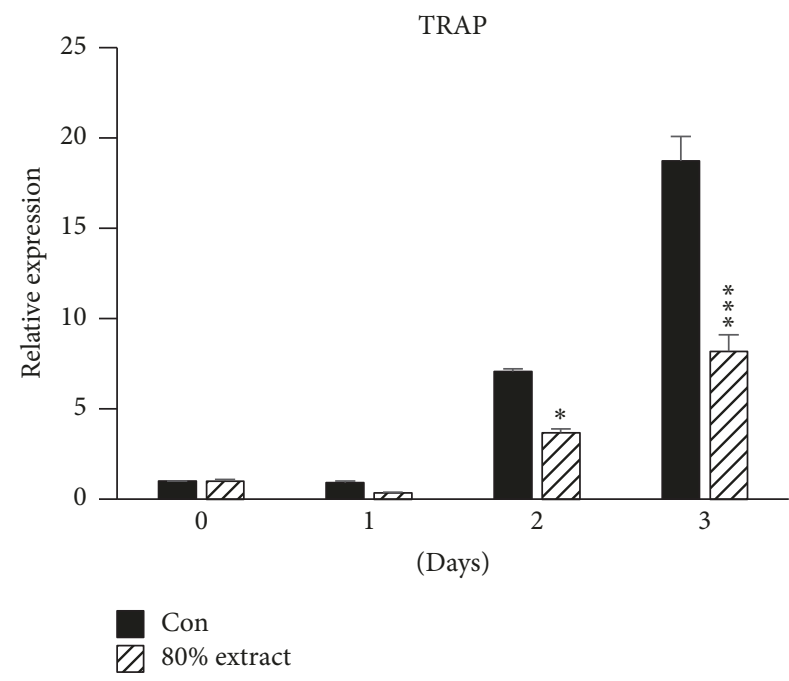

(d)

FiguRE 3: Effect of C. coreana Uyeki flos extracts on RANKL induced the mRNA expressions of osteoclastic-specific genes. The BMMs were pretreated with vehicle $(0.1 \%$ DMSO) or C. coreana Uyeki flos extracts $(30 \mu \mathrm{g} / \mathrm{mL})$ for $1 \mathrm{hr}$ and then treated with RANKL (10 ng/mL) and MCSF $(30 \mathrm{ng} / \mathrm{mL})$ for the designated time periods. The total RNA was extracted using TRIzol reagent, and mRNA expression levels of NFATcl, OSCAR, Cathepsin K, and TRAP were measured by real-time PCR. The internal control was used with GAPDH. ${ }^{*} P<0.05$; ${ }^{* *} P<0.01$; ${ }^{* * *} P<0.001$.

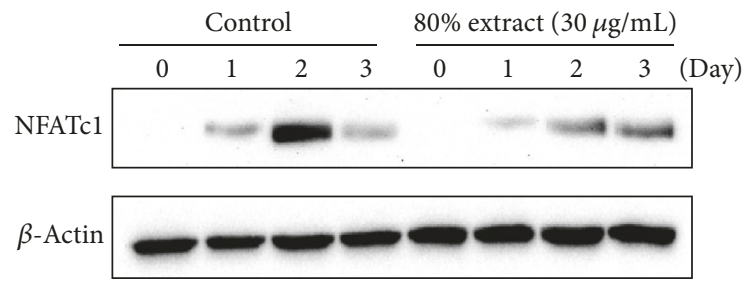

Figure 4: Effect of C. coreana Uyeki flos extracts on the translational expression of an osteoclast-specific transcription factor, NFATc1. BMMs were pretreated with vehicle $(0.1 \% \mathrm{DMSO})$ or C. coreana Uyeki flos extracts $(30 \mu \mathrm{g} / \mathrm{mL})$ for $1 \mathrm{hr}$ prior to RANKL $(10 \mathrm{ng} / \mathrm{mL})$ and M-CSF $(30 \mathrm{ng} / \mathrm{mL})$ treatment at the designated time periods. $\beta$-Actin was used as the internal control. of NF- $\kappa \mathrm{B}$ activation [18]. Previous studies have reported that QCT increased bone formation marker, such as osteocalcin. Derakhshanian et al. suggested that quercetin prevented the glucocorticoid-induced osteoporosis by its bone enhancing effect [19]. Therefore, QCT is a potential substance in the extract of $C$. coreana that induces the activity of antiosteoclast and proosteoblast. The antiosteoclastic activity of QCT by osteoclast differentiation in vitro assay in our lab (data not shown) was confirmed. Satué et al. reported that QCIT promotes osteoblastogenesis in MC3T3-E1 cells and inhibits osteoclast differentiation in RAW264.7 cells, highlighting the positive effect of QCIT on the prevention of osteoporosis [20]. 


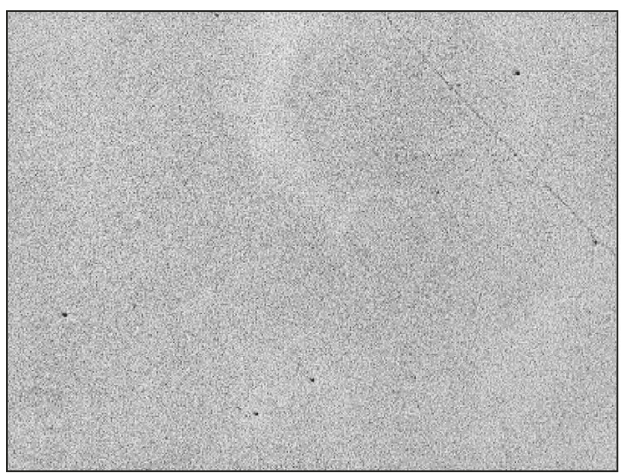

Negative

(a)

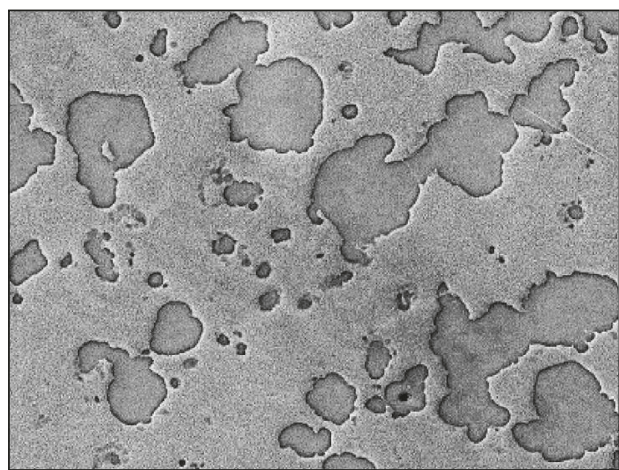

$10 \mu \mathrm{g} / \mathrm{mL}$

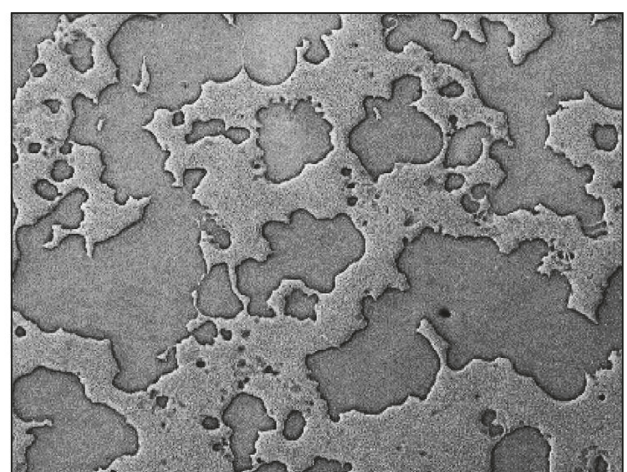

Positive

(b)

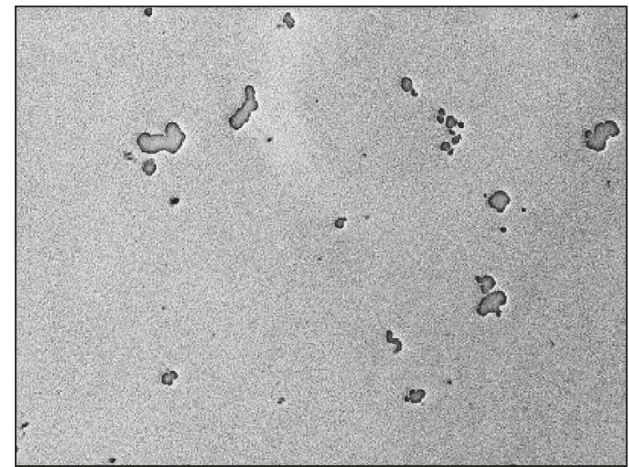

$30 \mu \mathrm{g} / \mathrm{mL}$

$80 \%$ extract of C. coreana Uyeki flos

(c) (d)

Figure 5: Resorption pit assay for osteoclast. The BMMs were plated at $6 \times 10^{6}$ cells/well in a 24-well tissue culture plate and cultured with the conditions of $30 \mathrm{ng} / \mathrm{mL}$ M-CSF in a negative/positive (without/with $10 \mathrm{ng} / \mathrm{mL}$ RANKL) ((a) and (b), resp.) and the presence (10 and $30 \mu \mathrm{g} / \mathrm{mL}$, (c) and (d), resp.) of $80 \%$ C. coreana Uyeki flos for 4 days.

Several proteins are involved in the functioning of osteoclast differentiation. Some of the important proteins were analyzed by real-time qPCR. The OSCAR presents a costimulatory receptor that is important for osteoclast differentiation and is involved in the activation of NFATcl and macrophage immune cell regulators [21]. Therefore, OSCAR is associated with osteoporosis and rheumatoid arthritis and may trigger their onset mechanism [22, 23]. Cathepsin $\mathrm{K}$ is expressed mainly in osteoclasts and acts as a lysosomal cysteine protease [24]. The protein also plays a significant role in degrading the organic composition of bone (predominantly Type I collagen) [25]. Cathepsin K plays a significant role in bone resorptive mechanism. Odanacatib (ODN), an inhibitor of cathepsin $\mathrm{K}$, is currently being developed as a treatment for osteoporosis [26]. TRAP is expressed strongly in osteoclastic cell and induces osteoclast differentiation $[27,28]$. TRAP is involved in osteoclast migration to the spot of bone resorption and begins osteoclast differentiation [29]. In this study, TRAP, OSCAR, and cathepsin K, which are important mediators of osteoclast differentiation, were inhibited by the treatment with $C$. coreana Uyeki flos.
Interestingly, unlike the expression pattern of increasing expression of NFATc1 mRNA in the presence of RANKL until day 3, the level of translational expression of NFATc1 peaked at day 2 and rapidly decreased to baseline by day 3. Because of the nuclear import of these proteins in the presence of RANKL, the expression level of NFATcl protein was decreased during late-stage osteoclast differentiation by an auto-feedback system of NFATc1 but the mRNA expression level of NFATc1 is maintained until the last stage of osteoclastogenesis [30].

\section{Conclusions}

In the present study, the alcoholic extracts of C. coreana Uyeki flos were prepared, and the ethanol extraction process was optimized with respect to the total flavonoid, total phenolic content, contents of active compounds, and antiosteoclastic activity. The in vitro study showed that the optimized $80 \%$ alcoholic extract could inhibit osteoclast differentiation significantly at a concentration of $30 \mu \mathrm{g} / \mathrm{mL}$. In addition, the osteoclast inhibited by $C$. coreana Uyeki flos had a resorptive activity; that is, the resorptive activity 
was suppressed by the extract of $C$. coreana Uyeki flos. The extract of $C$. coreana Uyeki flos was analyzed by HPLC and its contents were identified. In vitro study showed that the antiosteoclastic activity of the extract was caused by total phenolics, flavonoids, and some phytochemicals, such as QCT and QCIT, synergistically. This paper is the first scientific report of the antiosteoclastic activity of $C$. coreana Uyeki flos extracts, highlighting the feasibility of the present herbal medicine for the treatment of osteoporosis.

\section{Conflicts of Interest}

The authors declare that there are no conflicts of interest.

\section{Acknowledgments}

This study was supported by Grants from National Research Foundation of Korea (NRF) (2016R1A6A3A11930984 and 2017R1C1B5015187) and the Forest Science \& Technology Projects (Project no. 2017024A00-1720-BA01) by Korea Forest Service.

\section{References}

[1] W. J. Boyle, W. S. Simonet, and D. L. Lacey, "Osteoclast differentiation and activation," Nature, vol. 423, no. 6937, pp. 337-342, 2003.

[2] M. Baud'huin, L. Duplomb, C. Ruiz Velasco, Y. Fortun, D. Heymann, and M. Padrines, "Key roles of the OPG-RANKRANKL system in bone oncology," Expert Review of Anticancer Therapy, vol. 7, no. 2, pp. 221-232, 2007.

[3] H. Takayanagi, "The role of NFAT in osteoclast formation," Annals of the New York Academy of Sciences, vol. 1116, pp. 227237, 2007.

[4] H. Takayanagi, S. Kim, T. Koga et al., "Induction and activation of the transcription factor NFATc1 (NFAT2) integrate RANKL signaling in terminal differentiation of osteoclasts," Developmental Cell, vol. 3, no. 6, pp. 889-901, 2002.

[5] M. H. Kim, S. Y. Ha, M. H. Oh, H. H. Kim, S. R. Kim, and M. W. Lee, "Anti-oxidative and anti-proliferative activity on human prostate cancer cells lines of the phenolic compounds from Corylopsis coreana Uyeki," Molecules, vol. 18, no. 5, pp. 48764886, 2013.

[6] H. Wang, G. J. Provan, and K. Helliwell, "Determination of hamamelitannin, catechins and gallic acid in witch hazel bark, twig and leaf by HPLC, Journal of Pharmaceutical and Biomedical Analysis, vol. 33, no. 4, pp. 539-544, 2003.

[7] J. Seo, J. Kim, J. Shim et al., "HPLC analysis, optimization of extraction conditions and biological evaluation of Corylopsis coreana uyeki flos," Molecules, vol. 21, no. 1, p. 94, 2016.

[8] I.-S. Yoon, D.-H. Park, S.-H. Ki, and S.-S. Cho, "Effects of extracts from Corylopsis coreana Uyeki (Hamamelidaceae) flos on xanthine oxidase activity and hyperuricemia," Journal of Pharmacy and Pharmacology, vol. 68, no. 12, pp. 1597-1603, 2016.

[9] T. Yin and L. Li, “The stem cell niches in bone," The Journal of Clinical Investigation, vol. 116, no. 5, pp. 1195-1201, 2006.

[10] A. Mansour, G. Abou-Ezzi, E. Sitnicka, S. E. W. Jacobsen, A. Wakkach, and C. Blin-Wakkach, "Osteoclasts promote the formation of hematopoietic stem cell niches in the bone marrow,"
The Journal of Experimental Medicine, vol. 209, no. 3, pp. 537549, 2012.

[11] J. Li, L. Zeng, J. Xie et al., "Inhibition of Osteoclastogenesis and Bone Resorption in vitro and in vivo by a prenylflavonoid xanthohumol from hops," Scientific Reports, vol. 5, Article ID 17605, 2015.

[12] H. Zheng, X. Yu, P. Collin-Osdoby, and P. Osdoby, "RANKL stimulates inducible nitric-oxide synthase expression and nitric oxide production in developing osteoclasts: An autocrine negative feedback mechanism triggered by RANKL-induced interferon- $\beta$ via NF- $\kappa \mathrm{B}$ that restrains osteoclastogenesis and bone resorption," The Journal of Biological Chemistry, vol. 281, no. 23, pp. 15809-15820, 2006.

[13] M. Matsumoto, T. Sudo, M. Maruyama, H. Osada, and M. Tsujimoto, "Activation of p38 mitogen-activated protein kinase is crucial in osteoclastogenesis induced by tumor necrosis factor," FEBS Letters, vol. 486, no. 1, pp. 23-28, 2000.

[14] F. Ikeda, R. Nishimura, T. Matsubara et al., "Critical roles of c-Jun signaling in regulation of NFAT family and RANKLrequlated osteoclast differentiation," The Journal of Clinical Investigation, vol. 114, no. 4, pp. 475-484, 2004.

[15] J.-Y. Choe and S.-K. Kim, "Melittin inhibits osteoclast formation through the downregulation of the RANKL-RANK signaling pathway and the inhibition of interleukin- $1 \beta$ in murine macrophages," International Journal of Molecular Medicine, vol. 39, no. 3, pp. 539-548, 2017.

[16] J. An, H. Yang, Q. Zhang et al., "Natural products for treatment of osteoporosis: the effects and mechanisms on promoting osteoblast-mediated bone formation," Life Sciences, vol. 147, pp. 46-58, 2016.

[17] N. Nazir, S. Koul, M. A. Qurishi et al., "Immunomodulatory effect of bergenin and norbergenin against adjuvant-induced arthritis-a flow cytometric study," Journal of Ethnopharmacology, vol. 112, no. 2, pp. 401-405, 2007.

[18] T. Oliveira, C. A. Figueiredo, C. Brito et al., “Allium cepa L. and quercetin inhibit RANKL/ porphyromonas gingivalis LPSinduced osteoclastogenesis by downregulating NF- $\kappa \mathrm{B}$ signaling pathway," Evidence-Based Complementary and Alternative Medicine, vol. 2015, Article ID 704781, 11 pages, 2015.

[19] H. Derakhshanian, M. Djalali, A. Djazayery et al., "Quercetin prevents experimental glucocorticoid-induced osteoporosis: a comparative study with alendronate," Canadian Journal of Physiology and Pharmacology, vol. 91, no. 5, pp. 380-385, 2013.

[20] M. Satué, M. del Mar Arriero, M. Monjo, and J. M. Ramis, "Quercitrin and taxifolin stimulate osteoblast differentiation in MC3T3-E1 cells and inhibit osteoclastogenesis in RAW 264.7 cells," Biochemical Pharmacology, vol. 86, no. 10, pp. 1476-1486, 2013.

[21] T. Koga, M. Inui, K. Inoue et al., "Costimulatory signals mediated by the ITAM motif cooperate with RANKL for bone homeostasis," Nature, vol. 428, no. 6984, pp. 758-763, 2004.

[22] L. C. Hofbauer and M. Schoppet, "Clinical implications of the osteoprotegerin/RANKL/RANK system for bone and vascular diseases," The Journal of the American Medical Association, vol. 292, no. 4, pp. 490-495, 2004.

[23] J. M. Weiss, A. C. Renkl, C. S. Maier et al., "Osteopontin is involved in the initiation of cutaneous contact hypersensitivity by inducing Langerhans and dendritic cell migration to lymph nodes," The Journal of Experimental Medicine, vol. 194, no. 9, pp. 1219-1229, 2001.

[24] D. Bromme and K. Okamoto, "Human Cathepsin 02, a novel cysteine protease highly expressed in osteoclastomas and ovary 
molecular cloning, sequencing and tissue distribution," Biological Chemistry Hoppe-Seyler, vol. 376, no. 6, pp. 379-384, 1995.

[25] W. Kafienah, D. Brömme, D. J. Buttle, L. J. Croucher, and A. P. Hollander, "Human cathepsin K cleaves native type I and II collagens at the N-terminal end of the triple helix," Biochemical Journal, vol. 331, part 3, pp. 727-732, 1998.

[26] S. Boonen, E. Rosenberg, F. Claessens, D. Vanderschueren, and S. Papapoulos, "Inhibition of cathepsin $\mathrm{K}$ for treatment of osteoporosis," Current Osteoporosis Reports, vol. 10, no. 1, pp. 7379, 2012.

[27] M. Sapkota, L. Li, H. Choi, W. H. Gerwick, and Y. Soh, "Bromohonaucin A inhibits osteoclastogenic differentiation in RAW 264.7 cells via Akt and ERK signaling pathways," European Journal of Pharmacology, vol. 769, pp. 100-109, 2015.

[28] J. E. Otero, S. Dai, M. A. Alhawagri, I. Darwech, and Y. AbuAmer, "IKK $\beta$ activation is sufficient for RANK-independent osteoclast differentiation and osteolysis," Journal of Bone and Mineral Research, vol. 25, no. 6, pp. 1282-1294, 2010.

[29] T.-J. Sheu, E. M. Schwarz, D. A. Martinez et al., "A phage display technique identifies a novel regulator of cell differentiation," The Journal of Biological Chemistry, vol. 278, no. 1, pp. 438-443, 2003.

[30] J. H. Kim, K. Kim, H. M. Jin et al., "Negative feedback control of osteoclast formation through ubiquitin-mediated downregulation of NFATcl," The Journal of Biological Chemistry, vol. 285, no. 8, pp. 5224-5231, 2010. 


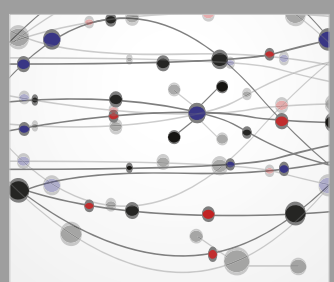

The Scientific World Journal
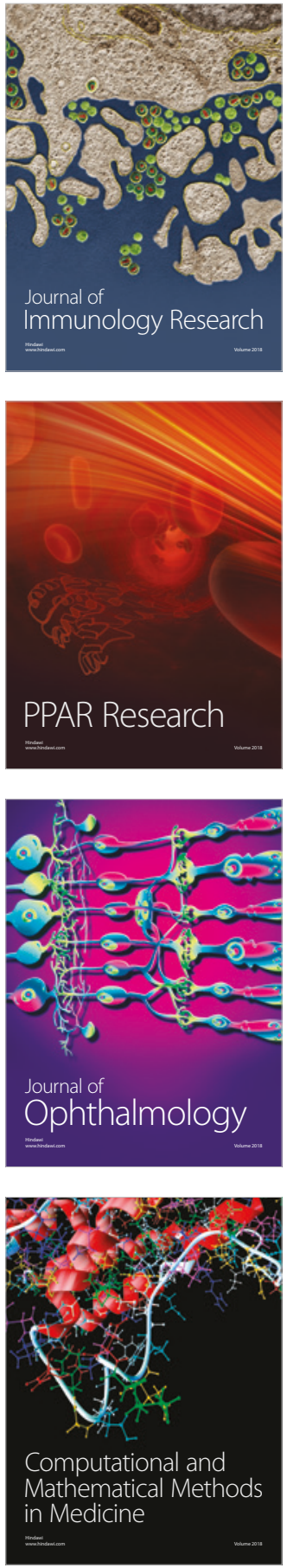

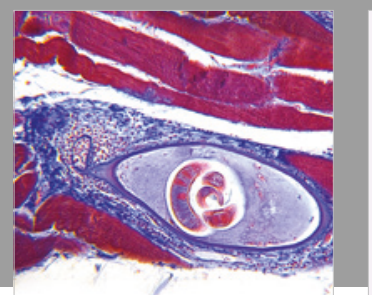

Gastroenterology Research and Practice

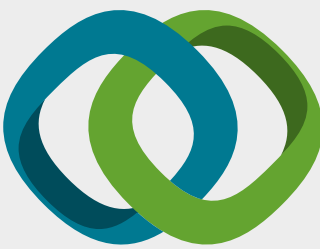

\section{Hindawi}

Submit your manuscripts at

www.hindawi.com
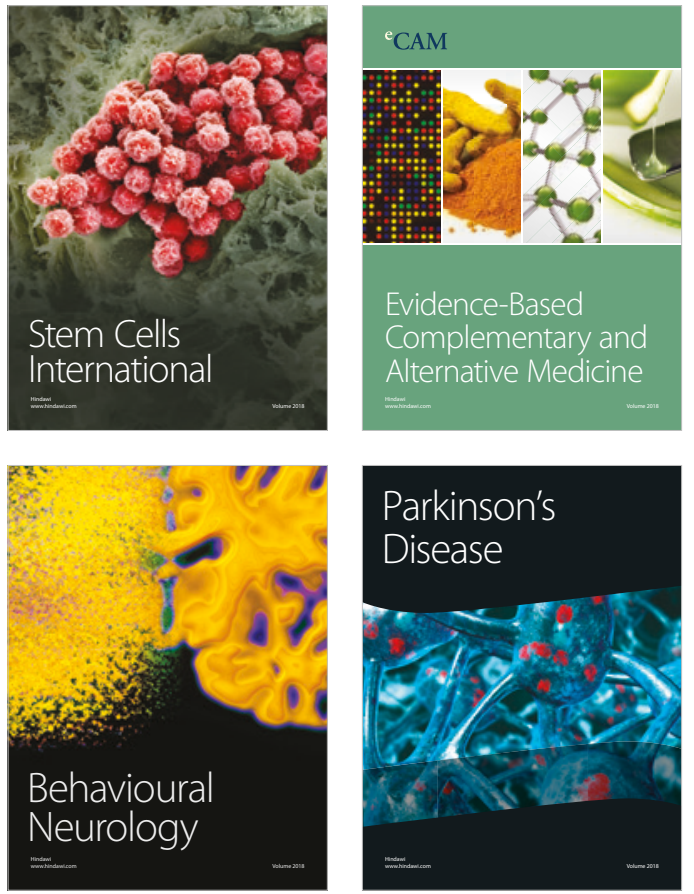

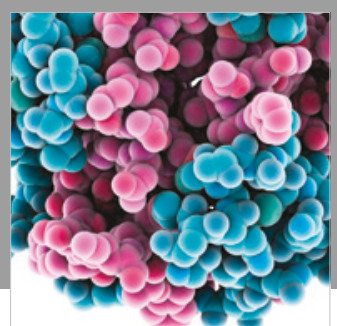

ournal of

Diabetes Research

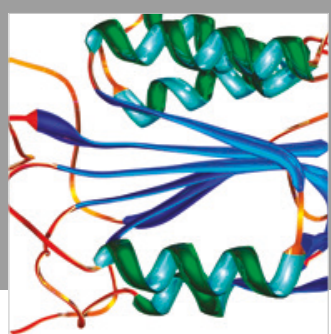

Disease Markers
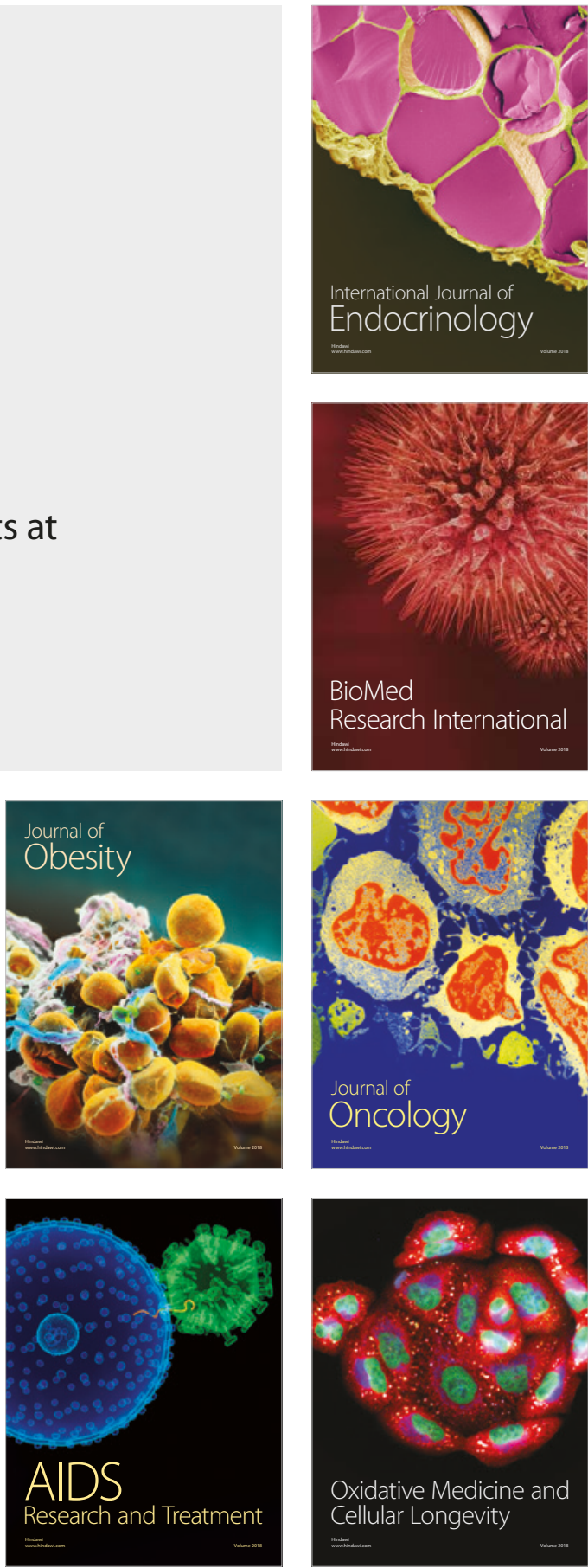